\title{
Om diagnostic applied to scalar field models and slowing down of cosmic acceleration
}

\author{
M. Shahalam ${ }^{1 \star}$, Sasha Sami ${ }^{2} \dagger$, Abhineet Agarwal $^{2} \ddagger$ \\ ${ }^{1}$ Center for Theoretical Physics, Jamia Millia Islamia, New Delhi-110025, India \\ ${ }^{2}$ International Institute of Information Technology Gachibowli, Hyderabad 500032, Telangana, India
}

5 October 2018

\begin{abstract}
We apply the $O m$ diagnostic to models for dark energy based on scalar fields. In case of the power law potentials, we demonstrate the possibility of slowing down the expansion of the Universe around the present epoch for a specific range in the parameter space. For these models, we also examine the issues concerning the age of Universe. We use the $\mathrm{Om}$ diagnostic to distinguish the $\Lambda \mathrm{CDM}$ model from non minimally coupled scalar field, phantom field and generic quintessence models. Our study shows that the $O m$ has zero, positive and negative curvatures for $\Lambda \mathrm{CDM}$, phantom and quintessence models respectively. We use an integrated data base $(\mathrm{SN}+\mathrm{Hubble}+\mathrm{BAO}+\mathrm{CMB})$ for observational analysis and demonstrate that $\mathrm{Om}$ is a useful diagnostic to apply to observational data.
\end{abstract}

Key words: cosmological parameters- cosmology: observations- cosmology: theory- dark energy.

\section{INTRODUCTION}

A number of observational investigations such as, Type Ia supernovae (Riess et al. 1998; Perlmutter et al. 1999), cosmic microwave background radiation (Spergel et al. 2003; Komatsu et al. 2011), surveys of the large scale structure (Eisenstein et al. 2005) and PLANCK 2013 (Ade et al. 2013) indicate that our Universe is accelerating at present. In the standard framework based upon Einstein gravity, cosmic acceleration can be explained by an exotic fluid with large negative pressure filling the Universe, dubbed 'dark energy' (Riess et al. 1998; Perlmutter et al. 1999; Sahni \& Starobinsky 2000; Spergel et al. 2003; Copeland, Sami \& Tsujikawa 2006; Sami 2009; Komatsu et al. 2011; Ade et al. 2013; Sami \& Myrzakulov 2013). The simplest candidate for dark energy (DE) - the cosmological constant $\Lambda$ is plagued with difficult theoretical issues related to fine tuning. A variety of other dark energy models have been studied in the literature, namely, quintessence (Ratra \& Peebels 1988), $\Lambda$ CDM (Weinberg 1989) and the phantom field (Caldwell, Kamionkowski \& Weinberg 2003; Setare 2007).

Alternatively, cosmic acceleration can also be mimicked by the large scale modification of gravity (Boisseau et al. 2000; Dvali, Gabadadze \& Porrati 2000; Sahni \& Shtanov 2003; Sahni, Shtanov \& Viznyuk 2005; Trodden 2007; Brevik 2008; Ali, Gannouji \& Sami 2010; Gannouji \& Sami

\footnotetext{
* mohdshahamu@gmail.com

† sashasami03@gmail.com

‡ agarwal.abhi93@gmail.com
}

2010). Though, the late time cosmic acceleration is considered to be an established phenomenon at present, its underlying cause remains uncertain. A large number of models, within the framework of standard lore and modified theories of gravity can explain the said phenomenon. It is therefore important to find ways to discriminate between various competing models. To this effect, important geometrical diagnostics have been recently suggested in the literature such as, Statefinder (Alam et al. 2003; Sahni et al. 2003), Statefinder hierarchy (Arabsalmani \& Sahni 2011), Om (Sahni, Shafieloo \& Starobinsky 2008, 2014) and Om3 (Shafieloo, Sahni \& Starobinsky 2012). Statefinders use the second, third and higher order derivatives of the scale factor with respect to cosmic time whereas $\mathrm{Om}$ relies on first order derivative alone. Consequently $\mathrm{Om}$ is a simpler diagnostic when applied to observations.

The statefinder method has been extensively used in the literature to distinguish among various models of dark energy and modified theories of gravity. For instance, non minimally coupled scalar field, galileon field, Dvali, Gabadadze and Porrati (DGP) model, bimetric (bigravity) theory of massive gravity and others have been investigated (Sami et al. 2012; Myrzakulov \& Shahalam 2013) using this diagnostic. Observational constraints have been put on the statefinder pair $\{r, s\}$ and deceleration parameter $q$ using Union2.1 compilation data (Suzuki et al. 2012) and 28 points of Hubble data (Farooq \& Ratra 2013), for the power law cosmological model (Rani et al. 2014). The statefinder analysis has been applied to non minimally coupled galileons 
and $f(T)$ cosmology by several authors (Jamil et al. 2012; Jamil, Momeni \& Myrzakulov 2013). Recently, statefinder hierarchy has been applied to models based upon Chaplygin gas, light mass galileons and holographic dark energy $(\mathrm{Li}$, Yang \& Chen 2014; Myrzakulov \& Shahalam 2014; Zhang, Cui \& Zhang 2014).

Coming back to $\mathrm{Om}$ diagnostic, to be employed in this paper, we should note its excellent features. First, it can discriminate dynamical dark energy models from $\Lambda$ CDM, in a robust way, even if the value of the matter density is not precisely known. Secondly, it can provide a null test of $\Lambda$ CDM hypothesis, i.e., $O m(z)-\Omega_{0 m}=0$, if dark energy is a cosmological constant. $\mathrm{Om}$ has zero, negative and positive curvatures for $\Lambda \mathrm{CDM}$, quintessence and phantom models respectively.

Our paper is organised as follows: In section 2 we briefly revisit the $\mathrm{Om}$ diagnostic, to be used in the subsequent sections. In section 3 we display evolution equations of phantom (non-phantom) scalar field in the autonomous form. In sub-section 3.1. we consider power law potentials and examine the slowing down of cosmic acceleration for a possible range of the parametric space, whereas in sub-section 3.2 we consider age of the Universe in these models. In sub-section 3.3. we examine equation of state (EOS) and $\mathrm{Om}$ behaviour for the tracking potential $V(\phi)=V_{0}\left[\cosh \left(\tilde{\alpha} \phi / M_{p}\right)-1\right]^{p}$. Sub-section 3.4 and section 4 are devoted to application of $\mathrm{Om}$ to phantom field with linear potential and non minimally coupled scalar field respectively. Our results are summarized in the last section. In appendix A, we carry out joint data analysis and put observational constraints on the model parameters using Union2.1 compilation data (Suzuki et al. 2012), 28 points of Hubble data (Farooq \& Ratra 2013), BAO data (Eisenstein et al. 2005; Percival et al. 2010; Beutler et al. 2011; Blake et al. 2011; Jarosik et al. 2011; Giostri et al. 2012) and CMB data (Komatsu et al. 2011).

\section{Om DIAGNOSTIC}

$\mathrm{Om}$ is a geometrical diagnostic which combines Hubble parameter and redshift. It can differentiate a dynamical dark energy model from $\Lambda \mathrm{CDM}$, with and without reference to matter density. Constant behaviour of $\operatorname{Om}(z)$ with respect to $z$ signifies that DE is a cosmological constant $(\Lambda \mathrm{CDM})$. The positive slope of $O m(z)$ implies that dark energy is phantom $(w<-1)$ whereas the negative slope means that DE behaves like quintessence $(w>-1)$. Following Sahni et al. (2008); Zunckel \& Clarkson (2008), Om(z) for spatially flat Universe is defined as

$$
O m(z) \equiv \frac{H^{2}(z) / H_{0}^{2}-1}{(1+z)^{3}-1},
$$

where $H_{0}$ is the present value of the Hubble parameter. Since, for constant equation of state parameter,

$$
H^{2}(z)=H_{0}^{2}\left(\Omega_{0 m}(1+z)^{3}+\left(1-\Omega_{0 m}\right)(1+z)^{3(1+w)}\right),
$$

we have the following expression for $\operatorname{Om}(z)$,

$$
O m(z)=\Omega_{0 m}+\left(1-\Omega_{0 m}\right) \frac{(1+z)^{3(1+w)}-1}{(1+z)^{3}-1},
$$

which shows that, $\operatorname{Om}(z)=\Omega_{0 m}$ for $\Lambda \mathrm{CDM}$, whereas $O m(z)>\Omega_{0 m}$ for quintessence $(w>-1)$ and $O m(z)<\Omega_{0 m}$

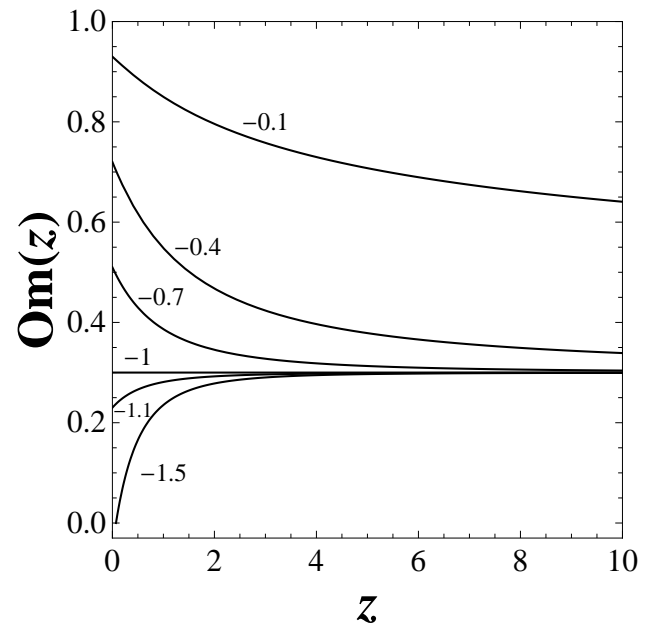

Figure 1. This figure shows the evolution of $\operatorname{Om}(z)$ versus the redshift $z$ for DE models with $\Omega_{0 m}=0.3$ and $w=-1.5,-1.1$, $-1,-0.7,-0.4,-0.1$ (lower to top). The horizontal line represents $\Lambda$ CDM with $w=-1$, and has zero curvature. The DE models with $w>-1$ (quintessence) have negative curvature whereas DE models with $w<-1$ (phantom) have positive curvature.

for phantom $(w<-1)$. This kind of behaviour can be clearly seen in figure 1 It is remarkable that $\mathrm{Om}$ provides a null test of the $\Lambda$ CDM hypothesis. Another conclusion, that follows from this figure is that the growth of $\operatorname{Om}(z)$ at late time favours the decaying dark energy models (Shafieloo, Sahni \& Starobinsky 2009).

\section{SCALAR FIELD DYNAMICS}

In this section, we will examine cosmological dynamics of phantom (non-phantom) field with generic potentials, using $\mathrm{O} m$ diagnostic. We will be interested in distinguishing features of the dynamics which allow us to differentiate these models from $\Lambda$ CDM. Secondly, we will investigate the possibility of slowing down of cosmic acceleration in these models. The models showing transient acceleration have been studied previously (Felder et al. 2002; Kallosh et al. 2002; Alam, Sahni \& Starobinsky 2003; Frampton \& Takahashi 2003; Kallosh \& Linde 2003; Sahni \& Shtanov 2003).

In a spatially flat Freidmann- Lemaitre- RobertsonWalker (FLRW) background, the equations of motion for a scalar field take the form

$$
\begin{aligned}
3 M_{\mathrm{pl}}^{2} H^{2} & =\rho_{m}+\rho_{r}+\rho_{\phi}, \\
M_{\mathrm{pl}}^{2}\left(2 \dot{H}+3 H^{2}\right) & =-\frac{\rho_{r}}{3}-p_{\phi}, \\
\epsilon \ddot{\phi}+3 H \epsilon \dot{\phi}+\frac{d V(\phi)}{d \phi} & =0,
\end{aligned}
$$

where,

$$
\begin{aligned}
& \rho_{\phi}=\frac{1}{2} \epsilon \dot{\phi}^{2}+V(\phi), \\
& p_{\phi}=\frac{1}{2} \epsilon \dot{\phi}^{2}-V(\phi) ; \quad w_{\phi}=p_{\phi} / \rho_{\phi},
\end{aligned}
$$

and $\epsilon=+1$ and -1 corresponds to ordinary scalar and phantom field respectively. 

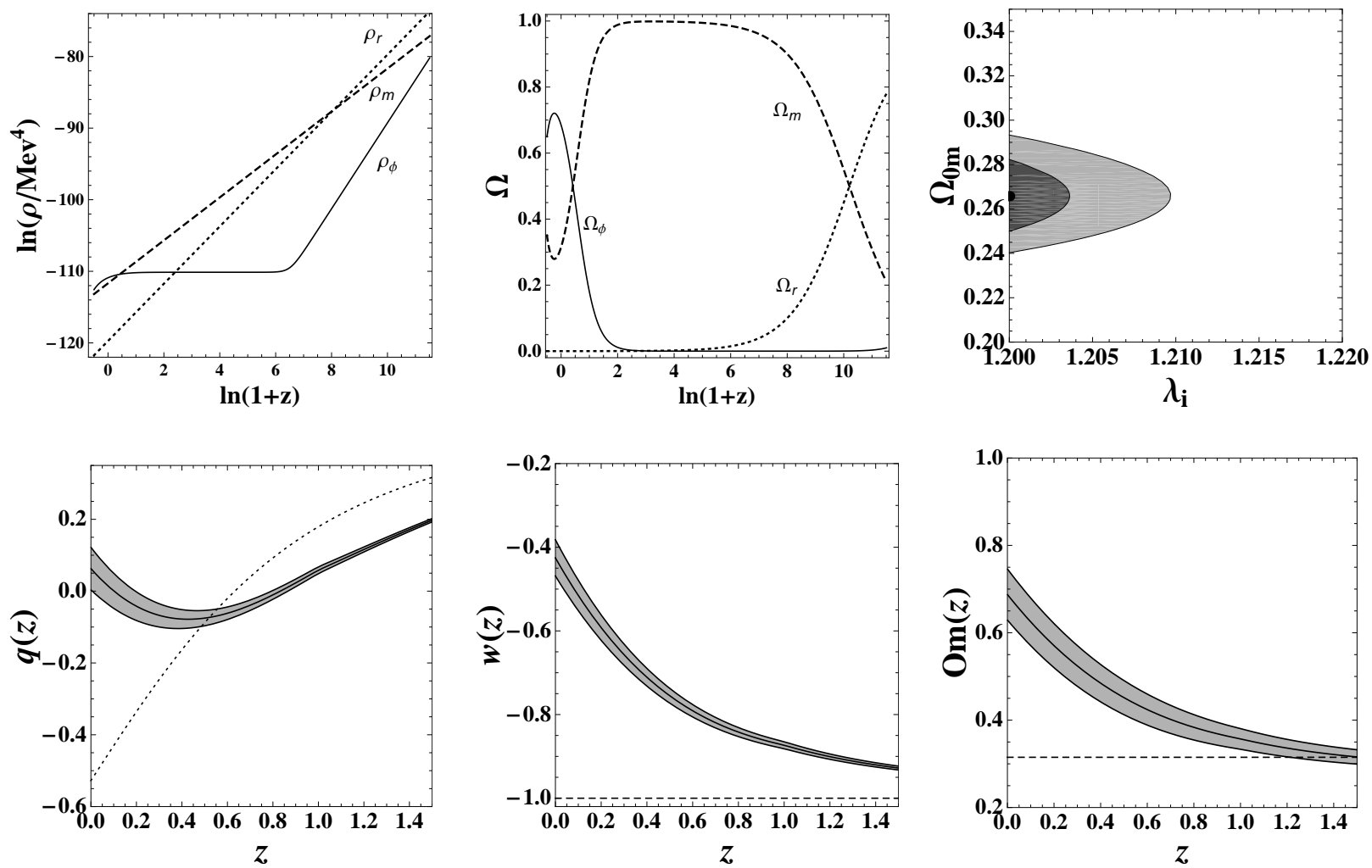

Figure 2. This figure shows different plots for the potential (16) with $m=1$. The upper left plot shows the evolution of energy density versus the redshift $z$. The dotted, dashed and black lines correspond to the energy density of radiation, matter and scalar field respectively. Initially, the energy density of scalar field $\left(\rho_{\phi}\right)$ is extremely sub-dominant and remains so, for most of the period of evolution. At late times, the field energy density catches up with the background and overtakes it. Around the present epoch, the field energy density starts decaying and correspondingly its equation of state starts approaching towards matter. The upper middle plot shows the evolution of density parameter $(\Omega)$ versus the redshift $z$. The upper right plot shows the $1 \sigma$ (dark shaded) and $2 \sigma$ (light shaded) likelihood contours in the $\lambda_{i}-\Omega_{0 m}$ plane, where $\lambda_{i}$ is the initial value of $\lambda$. The black dot designates the best fit value. The lower left plot shows the evolution of $q$ versus the redshift $z$. The value of $q$ increases at redshift $z \lesssim 0.4$ and becomes positive at late times. The lower middle and lower right plots show the evolution of $w(z)$ and $\operatorname{Om}(\mathrm{z})$ versus the redshift $z$. In all lower plots, dashed line represents $\Lambda$ CDM with $\Omega_{0 m}=0.315$; solid (middle) line inside shaded regions show best fitted behaviour and shaded regions show $1 \sigma$ confidence level. We have used joint data $(\mathrm{SN}+\mathrm{Hubble}+\mathrm{BAO}+\mathrm{CMB})$, see appendix A for details.

Let us introduce the following dimensionless quantities,

$$
x=\frac{\dot{\phi}}{\sqrt{6} H M_{\mathrm{pl}}}, \quad y=\frac{\sqrt{V}}{\sqrt{3} H M_{\mathrm{pl}}}, \quad \lambda=-M_{\mathrm{pl}} \frac{V^{\prime}}{V},
$$

which are used to form an autonomous system,

$$
\begin{aligned}
x^{\prime} & =x\left(\frac{\ddot{\phi}}{H \dot{\phi}}-\frac{\dot{H}}{H^{2}}\right), \\
y^{\prime} & =-y\left(\sqrt{\frac{3}{2}} \lambda x+\frac{\dot{H}}{H^{2}}\right), \\
\Omega_{r}^{\prime} & =-2 \Omega_{r}\left(2+\frac{\dot{H}}{H^{2}}\right), \\
\lambda^{\prime} & =\sqrt{6} x \lambda^{2}(1-\Gamma),
\end{aligned}
$$

where,

$$
\begin{aligned}
\frac{\dot{H}}{H^{2}} & =\frac{-3-3 \epsilon x^{2}+3 y^{2}-\Omega_{r}}{2}, \\
\frac{\ddot{\phi}}{H \dot{\phi}} & =\frac{-6 x+\sqrt{6} \epsilon y^{2} \lambda}{2 x}, \\
\Gamma & \equiv \frac{V V_{, \phi \phi}}{V_{, \phi}^{2}}
\end{aligned}
$$

and prime $\left({ }^{\prime}\right)$ denotes the derivative with respect to $\ln a$.

\subsection{Quintessence field and slowing down of cosmic acceleration}

In this sub-section, we shall consider scalar field models which can facilitate the slowing down of cosmic acceleration, at late times. Figure 1 shows that $\operatorname{Om}(z)$ increases with increasing constant equation of state, at late times. This effect could correspond to dark energy decaying into dark matter or something else. To check this possibility, let us consider 

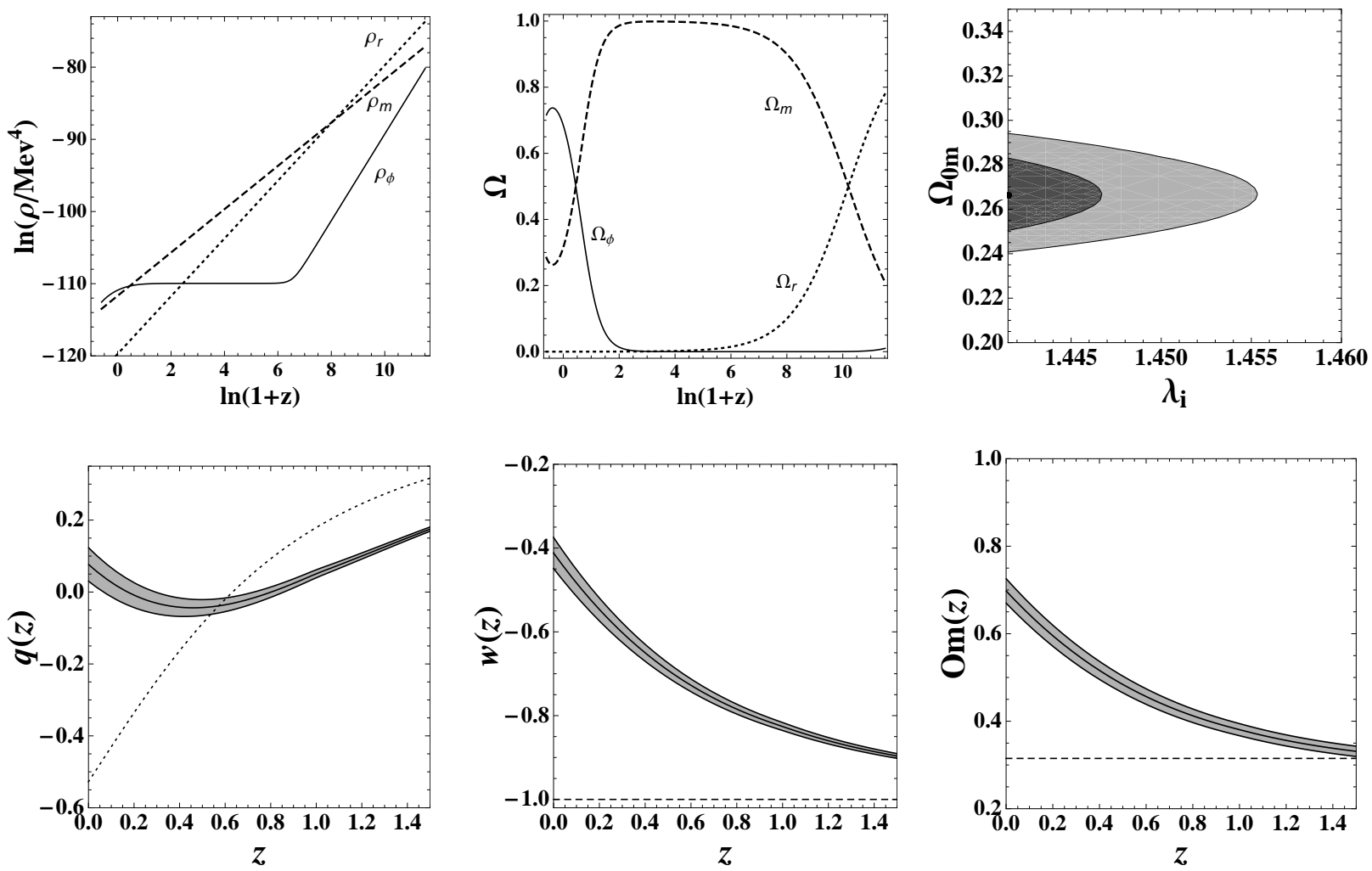

Figure 3. This figure is similar to figure 2 for the potential (16) but with $m=2$. The best fit values of the parameters are, $\lambda_{i}=1.4415$ and $\Omega_{0 m}=0.2663$, where $\lambda_{i}$ is the initial value of $\lambda$.

the following power law potentials,

$$
V(\phi)=V_{0} \phi^{2 m}, m=1,2
$$

In this case, we have slow roll regime followed by a fast roll and the oscillatory phase thereafter as $\phi$ approaches the origin. The average equation of state during oscillations is given by $\langle w\rangle=(m-1) /(m+1)$ and therefore, $\langle w\rangle=0,1 / 3$ for $m=1,2$ respectively. In this case, if we set parameters such that evolution of Universe corresponds to slow roll regime in the recent past, then acceleration would register its slow down around the present epoch. Keeping this in mind, we evolve the equations of motion numerically. Our results are displayed in figures 2 and 3 In figure 2 the upper right plot shows the $1 \sigma$ (dark shaded) and $2 \sigma$ (light shaded) likelihood contours in the $\lambda_{i}-\Omega_{0 m}$ plane, where $\lambda_{i}$ is the initial value of $\lambda$. The best fit values of the parameters are, $\lambda_{i}=1.2$ and $\Omega_{0 m}=0.2657$. The lower middle and right plots show the evolution of $w(z)$ and $\operatorname{Om}(z)$ versus the redshift $z$ respectively. We find that the EOS of dark energy grows at late times which is clearly shown by $O m$ and $q$ plots. This corresponds to an increase of $O m$ and $q$ at redshift $z \lesssim 0.4$. We have used, $\mathrm{SN}+\mathrm{Hubble}+\mathrm{BAO}+\mathrm{CMB}$ joint data for analysis (see appendix A). In the parametric space, shown in $\lambda_{i}-\Omega_{0 m}$ plane, we find that acceleration begins to slow down around $z \simeq 0.4$ followed by deceleration at present epoch (see the lower plots of figures 2 and 3 ). We also note from $\mathrm{Om}$ plot that $\operatorname{Om}(z)$ has negative curvature. The negative curvature of quintessence allows us to distinguish it from $\Lambda \mathrm{CDM}$ as well as from phantom dark energy irrespective of a given current value of the matter density.

\subsection{Slowing down of late time cosmic acceleration and age consideration}

The role of gravity in the Universe filled with standard matter is to decelerate the expansion such that larger is the matter density, faster will be the expansion rate thereby smaller would be the age of Universe. In the standard lore, the only way to circumvent the age problem is provided by invoking a repulsive effect which could be described by a positive cosmological constant or by a slowly rolling scalar field. The repulsive effect becomes important at late times giving rise to large contribution to the age of Universe. It is therefore not surprising that more than half of the contribution to the age of Universe comes from the redshift in the interval $z \in(0,1)$, see figure 4 . The slowing down of acceleration also takes place in the said interval for chosen set of model parameters. This should clearly decrease the age of Universe. Then the requirement of consistency with data can only be achieved by adjusting the matter density parameter $\Omega_{0 m}$ which in a sense quantifies the attractive effect of gravity. Indeed, in case of $\phi^{2}$ potential, for which slowing down commences around $z \simeq 0.4$, the best fit value of the matter density parameter is, $\Omega_{0 m}=0.2657$ which is smaller than its $\Lambda$ CDM value quoted by the Planck (see Table 1). Let us also note that the best fit value of matter density parameter for the model $\left(w(z)=-\frac{1+\tanh \left[\left(z-z_{t}\right) \Delta\right]}{2}\right)$ given by Shafieloo 


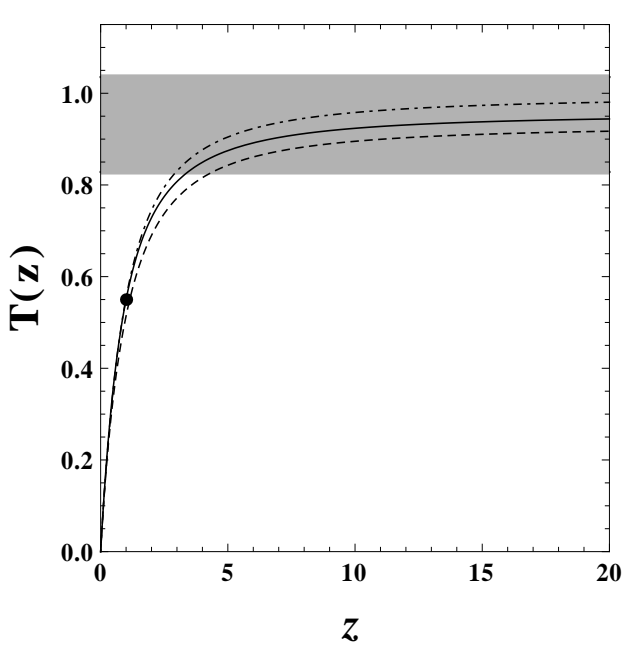

Figure 4. The dimensionless $T(z)=H_{0} t(z)(t(z)$ is the cosmic age at redshift $z$ ) is plotted for three models, namely, $\Lambda \mathrm{CDM}$ (solid line), quintessence with $\phi^{2}$ potential (dashed line) and the cosmological model with given equation of state, $w(z)=$ $-\frac{1+\tanh \left[\left(z-z_{t}\right) \Delta\right]}{2}$ (dot dashed line) which has same number of free parameters as the CPL ansatz but does not permit the crossing to phantom divide at $w=-1$. The form of $w(z)$ has been used (Shafieloo et al. 2009) to show slowing down of cosmic acceleration at $z \lesssim 0.3$. The best fit value of $\Omega_{0 m}=0.3086$ for $\Lambda \mathrm{CDM}$, is taken from Planck data result 2013, and the best fit values of the model parameters of $\phi^{2}$ potential are found to be $\lambda_{i}=1.2$ and $\Omega_{0 m}=0.2657$, (where $\lambda_{i}$ is the initial value of $\lambda$ ) whereas the best fit values of parameters for the ansatz are, $z_{t}=0.008, \Delta=12.8$ and $\Omega_{0 m}=0.255$ (Shafieloo et al. 2009). The horizontal band represents the range of the age of Universe based upon observations on globular clusters (Krauss \& Chaboyer 2003; Frieman, Turner \& Huterer 2008). The black dot designates the numerical value of $T(z)$ at $z=1(T(z=1)=0.5498)$.

Table 1. Age summary.

\begin{tabular}{cccc}
\hline \hline Data/Model & $\Omega_{0 m}$ & $\begin{array}{c}H_{0} \\
(\mathrm{Km} / \mathrm{s} / \mathrm{Mpc})\end{array}$ & $\begin{array}{c}t_{0} \\
(\text { by })\end{array}$ \\
\hline Planck & 0.3086 & 67.77 & 13.7965 \\
\hline$\Lambda$ CDM & 0.3086 & 67.77 & 13.8567 \\
\hline$\phi^{2}$ potential & 0.2657 & 67.77 & 13.4825 \\
\hline$w(z)=-\frac{1+\tanh \left[\left(z-z_{t}\right) \Delta\right]}{2}$ & 0.255 & 67.77 & 14.4039 \\
\hline \hline
\end{tabular}

et. al (2009), is smaller than its counter part obtained in $\phi^{2}$ model giving rise to some what larger value of the age (see figure 4).

The cosmic age $t(z)$ is defined as the cosmic time, Universe spends from a given $z$ to the present epoch as

$$
t(z)=\int_{0}^{z} \frac{d z^{\prime}}{\left(1+z^{\prime}\right) H\left(z^{\prime}\right)}
$$

where $H(z)$ is the Hubble parameter expressed in terms of redshift $z$. For spatially flat Universe, Hubble parameter can be written as

$$
\begin{aligned}
H(z)= & H_{0}\left[\Omega_{0 m}(1+z)^{3}+\left(1-\Omega_{0 m}\right)\right. \\
& \left.\exp \left(3 \int_{0}^{z}\left[1+w\left(z^{\prime}\right)\right] \frac{d z^{\prime}}{1+z^{\prime}}\right)\right]^{1 / 2}
\end{aligned}
$$

where $w=-1$ for $\Lambda$ CDM, $w(z)=\frac{\frac{1}{2} \dot{\phi}^{2}-V(\phi)}{\frac{1}{2} \dot{\phi}^{2}+V(\phi)}$ for quintessence with $\phi^{2}$ potential and $w(z)=-\frac{1+\tanh \left[\left(z-z_{t}\right) \Delta\right]}{2}$ for the cosmological model suggested by Shafieloo et al. (2009).

It is helpful to use a dimensionless cosmic age $T(z)=$ $H_{0} t(z)$, where $t(z)$ is given by equation (17) such that $T(z)=H_{0} t(z) \rightarrow 0$ as $z \rightarrow 0$ implying (a) $t(0) \rightarrow 0$, (b) $t(0) \ll t(z>1)$ as shown in figure 4

\subsection{Tracker}

Scalar field models with tracker solutions are of great importance in cosmology. A tracker solution is such that, during radiation and matter era, the field mimics the backgroundscaling regime and at late times it exits to late time cosmic acceleration. Once the model parameters are fixed, the exit to dark energy behaviour remains independent for a wide variety of initial conditions. Here we shall consider the following potential (Sahni \& Wang 2000):

$$
V(\phi)=V_{0}\left[\cosh \left(\tilde{\alpha} \phi / M_{p}\right)-1\right]^{p}, \quad 0<p<1 / 2
$$

The above potential has asymptotic form

$$
V(\phi)=\frac{V_{0}}{2^{p}} e^{p \tilde{\alpha} \phi / M_{p}}, \quad p \tilde{\alpha}|\phi| / M_{p} \gg 1,
$$

and

$$
V(\phi)=\frac{V_{0}}{2^{p}}\left(\frac{\tilde{\alpha} \phi}{M_{p}}\right)^{2 p}, \quad p \tilde{\alpha}|\phi| / M_{p} \ll 1,
$$

where $p \tilde{\alpha}=\alpha$. The power law behaviour of (19) near the origin leads to oscillations of $\phi$ when it approaches the origin. To have viable thermal history of the Universe, we need to have $\Omega_{\phi}=\frac{3\left(1+w_{B}\right)}{\alpha^{2}}=$ constant $\leq 0.01$ (Ade et al. 2013) during the radiation dominated era, which implies $\alpha \geq 20$ (here $w_{B}$ represents background equation of state). In this case, the scalar field behaves like background matter, for most of the history of Universe and, only at late times, it exits to dark energy which demands for tracker solution.

The potential under consideration has peculiarity near the origin for generic values of $p$. We have noticed that it is problematic to obtain oscillatory behaviour in the convex core of (19), using the autonomous system of equations. In what follows, we shall use the dimensionless form of evolution equations which allows us to control the dynamics of field, beginning from scaling regime to oscillations about $\phi=0$. The equations of motion have the following form,

$$
\begin{gathered}
\frac{\dot{a}^{2}(t)}{a^{2}(t)}=\frac{1}{3 M_{p}^{2}}\left[\rho_{\phi}+\rho_{m}+\rho_{r}\right] \\
\ddot{\phi}+3 \frac{\dot{a}}{a} \dot{\phi}+V_{, \phi}(\phi)=0 .
\end{gathered}
$$

In order to investigate the dynamics described by equations (22) and (23), it would be convenient to cast them as a system of first order equations

$$
\begin{gathered}
Y_{1}^{\prime}=\frac{Y_{2}}{h\left(Y_{1}, Y_{2}\right)} \\
Y_{2}^{\prime}=-3 Y_{2}-\frac{1}{h\left(Y_{1}, Y_{2}\right)}\left[\frac{d \mathcal{V}\left(Y_{1}\right)}{d Y_{1}}\right]
\end{gathered}
$$



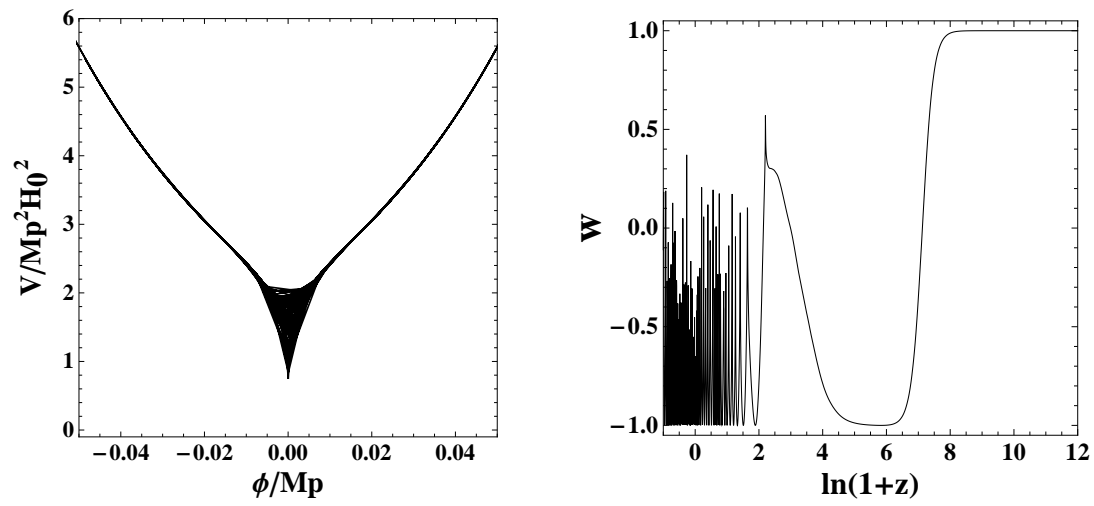

Figure 5. This figure shows the evolution of potential 19) and w versus field $\phi$ and redshift $z$ respectively. Figure shows that at late times the equation of state oscillates between zero and -1 such that the system spends most of the time around $\mathrm{w}=-1$. We have taken $\Omega_{0 m}=0.3, p=0.1, \tilde{\alpha}=200(\alpha=p \tilde{\alpha}=20)$ and $V_{0}=2.2 M_{p}^{2} H_{0}^{2}$.
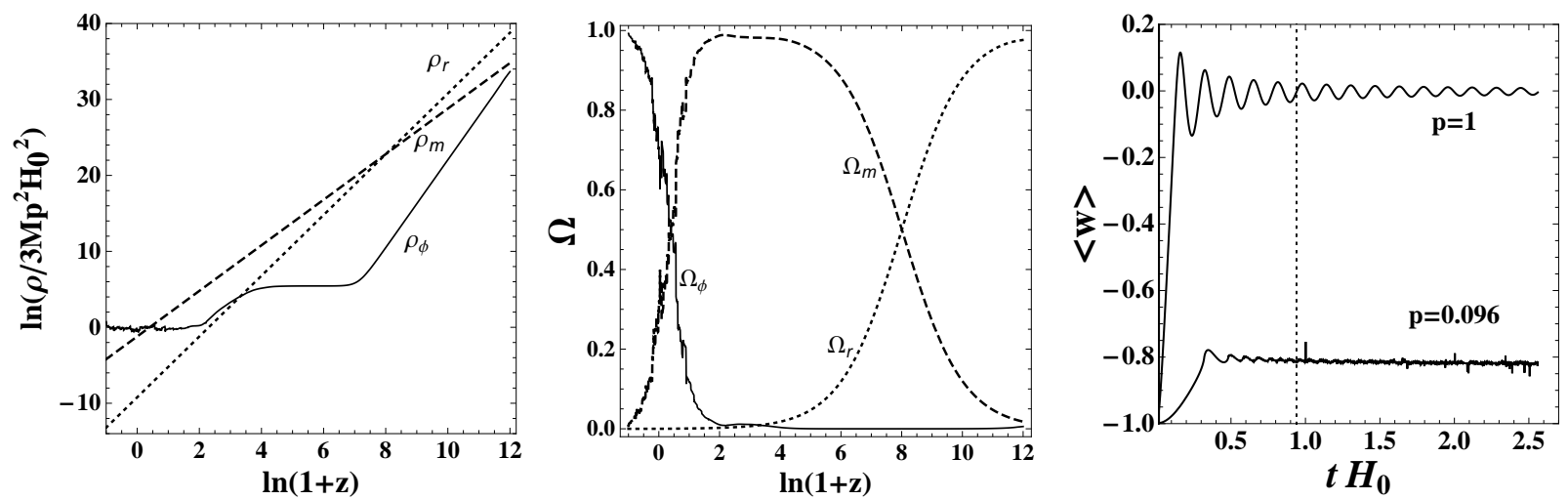

Figure 6. This figure shows different plots for the potential (19). The left and middle plots show the tracking behaviour of scalar field energy density and evolution of density parameter versus the redshift $z$. The dotted, dashed and black lines correspond to the radiation, matter and scalar field respectively. In both the plots the parameters are same as in figure 5 The right plot shows the evolution of average equation of state $\langle\mathrm{w}>$ versus cosmic time which agrees with $<\mathrm{w}\rangle=\frac{p-1}{p+1}$, our plot corresponds to $p=1, \Omega_{0 m}=0.319, \tilde{\alpha}=13$, $V_{0}=2.2 M_{p}^{2} H_{0}^{2}$ and $p=0.096, \Omega_{0 m}=0.319, \tilde{\alpha}=37.59, V_{0}=2.2 M_{p}^{2} H_{0}^{2}$. The vertical dotted line designates the present epoch.
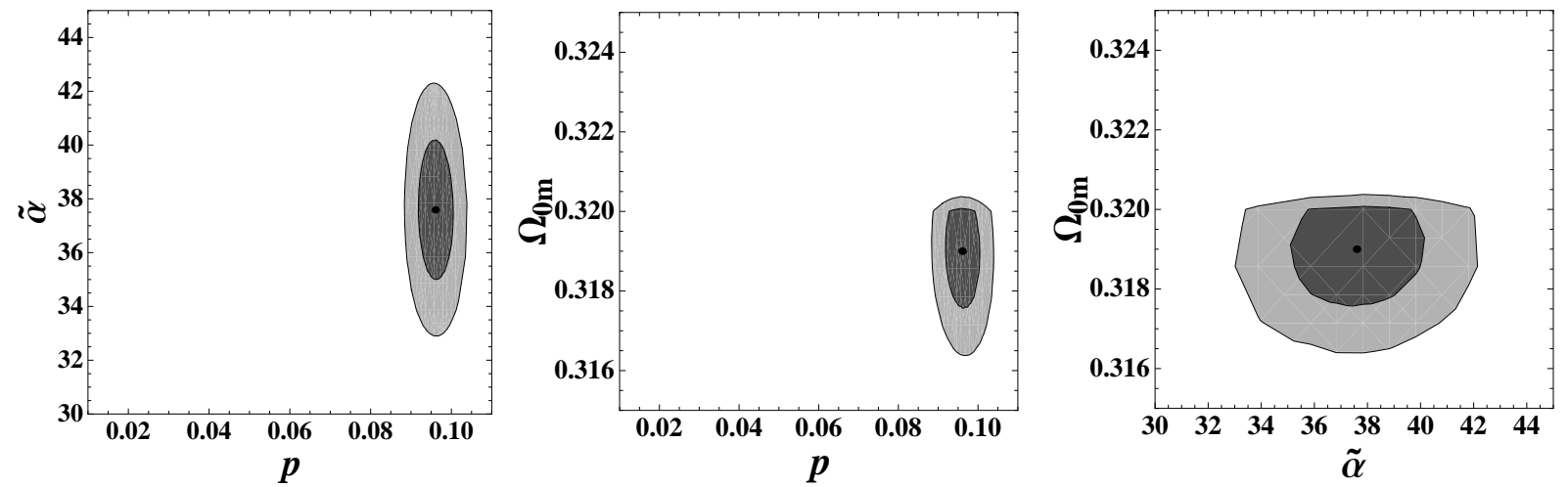

Figure 7. This figure shows the $1 \sigma$ (dark shaded) and $2 \sigma$ (light shaded) likelihood contours for the potential 19) with joint data $(\mathrm{SN}+\mathrm{Hubble}+\mathrm{BAO}+\mathrm{CMB})$; black dots designate the best fit value of the parameters which are found to be $\Omega_{0 m}=0.319, p=0.096$ and $\tilde{\alpha}=37.59$. 

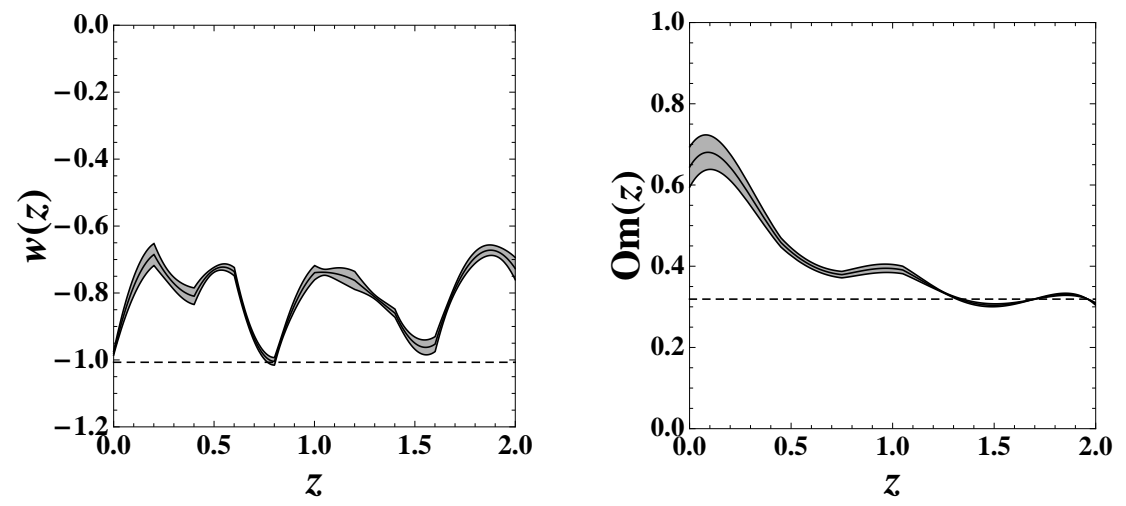

Figure 8. This figure shows the evolution of $w(z)$ and $\operatorname{Om}(z)$ versus the redshift $z$ for the potential (19). Equation of state exhibits oscillating behaviour which has also been manifested in $\operatorname{Om}(z)$ plot. In both the plots, dashed line represents $\Lambda$ CDM with $\Omega_{0 m}=0.319$; solid (middle) lines inside shaded regions show best fitted behaviour and shaded regions show $1 \sigma$ confidence level. We have used joint data $(\mathrm{SN}+\mathrm{Hubble}+\mathrm{BAO}+\mathrm{CMB})$ in our analysis.

where

$$
Y_{1}=\frac{\phi}{M_{p}}, \quad Y_{2}=\frac{\dot{\phi}}{M_{p} H_{0}}, \quad \mathcal{V}=\frac{V\left(Y_{1}\right)}{M_{p}^{2} H_{0}^{2}}
$$

and prime denotes the derivative with respect to the variable $N=\ln (a)$. The function $h\left(Y_{1}, Y_{2}\right)$ is given as:

$$
h\left(Y_{1}, Y_{2}\right)=\sqrt{\left[\frac{Y_{2}^{2}}{6}+\frac{\mathcal{V}\left(Y_{1}\right)}{3}+\Omega_{0 m} e^{-3 a}+\Omega_{0 r} e^{-4 a}\right]}
$$

where $\Omega_{0 m}$ and $\Omega_{0 r}$ are the present energy density parameters of matter and radiation respectively. We have numerically solved the evolution equations (24) \& (25); Our results are shown in figures [5, 6, 7 and 8 Figure 5 shows the behaviour of the potential (19) versus field $\phi$ and the equation of state parameter $\mathrm{w}$ versus the redshift $z$. As the field evolves from the steep region towards the origin, $\rho_{\phi}$ undershoots the background and field freezes for a while due to Hubble damping (see the left and the middle plots of figure 6). The field then approximately mimics the background before approaching the convex core of the potential, where oscillations set in. It is clear from the right plot of figure 5 that field oscillates most of the time near $\mathrm{w}=-1$. The right plot of figure 6 shows average value of $\mathrm{w}$ versus the cosmic time for $p=1 \& p=0.096$ which agrees with the analytical result, $<\mathrm{w}\rangle=p-1 / p+1$ at the attractor point.

In figure [7, all plots show the $1 \sigma$ (dark shaded) and $2 \sigma$ (light shaded) likelihood contours. Figure 8 shows the oscillating behaviour of equation of state and the corresponding behaviour of $\mathrm{Om}$ inside $1 \sigma$ confidence level. The joint data $(\mathrm{SN}+\mathrm{Hubble}+\mathrm{BAO}+\mathrm{CMB})$ was used for carrying out the observational analysis (see appendix A). We should note that the best fit value for the parameter $p$ is different from $p=0$ that would correspond to the case of cosmological constant. The latter is reinforced by figure 8 which clearly shows, the model under consideration differs from $\Lambda$ CDM within $1 \sigma$ confidence level. In this case, we should further check for $\chi_{\text {red }}^{2}$. We find that the numerical value of $\chi_{\text {red }}^{2}=0.849713$ corresponding to the best fit value of $p$, is much smaller than one (see Table 2). Thus, we can not conclude that the underlying model is preferred over $\Lambda$ CDM.
Table 2. Comparison between $\chi_{\text {red }}^{2}$ values for the potential (19) and $\Lambda$ CDM. To obtain $\chi_{\text {red }}^{2}$ for the potential (19), we vary $p$ and put the best fit values of the remaining parameters, $\Omega_{0 m}=0.319$ and $\tilde{\alpha}=37.59$. The bold values correspond to the best fit value of $p$ and $\Omega_{0 m}$ for the potential (19) and $\Lambda$ CDM respectively.

\begin{tabular}{cl||cc}
\hline \hline \multicolumn{2}{c||}{ Potential (19) } & \multicolumn{2}{c}{$\Lambda \mathrm{CDM}$} \\
$p$ & $\chi_{\text {red }}^{2}$ & & $\Omega_{0 m}$ \\
& & & $\chi_{\text {red }}^{2}$ \\
\hline 0.01 & 0.951237 & 0.25 & 0.956061 \\
0.02 & 0.973990 & 0.26 & 0.948286 \\
0.03 & 0.987693 & 0.27 & 0.943602 \\
0.04 & 0.992347 & $\mathbf{0 . 2 8}$ & $\mathbf{0 . 9 4 1 7 4}$ \\
0.05 & 0.985540 & 0.29 & 0.942457 \\
0.06 & 0.972094 & 0.30 & 0.945545 \\
0.07 & 0.952011 & 0.31 & 0.950822 \\
0.08 & 0.888724 & 0.32 & 0.958119 \\
0.09 & 0.855366 & 0.33 & 0.967293 \\
$\mathbf{0 . 0 9 6}$ & $\mathbf{0 . 8 4 9 7 1 3}$ & 0.34 & 0.978213 \\
0.10 & 0.851936 & 0.35 & 0.990760 \\
\hline \hline
\end{tabular}

In this case, we could not find slowing down effect, for any values of the model parameters.

\subsection{Phantom field with linear potential}

Phantom dark energy with equation of state $w<-1$ can be achieved by introducing a negative kinetic energy term in the action of the scalar field. By putting $\epsilon=-1$ in equations (6) and (7), we get equation of motion, energy density and pressure of phantom field (Singh, Sami \& Dadhich 2003). In what follows, we shall examine the dynamic of a phantom field. In this case, the Hubble parameter for spatially flat Universe can be written as

$$
\begin{aligned}
H^{2}(z)= & H_{0}^{2}\left[\Omega_{0 r}(1+z)^{4}+\Omega_{0 m}(1+z)^{3}\right. \\
& \left.+\Omega_{0 \phi} \exp \left(3 \int_{0}^{z}\left[1+w_{\phi}\left(z^{\prime}\right)\right] \frac{d z^{\prime}}{1+z^{\prime}}\right)\right],
\end{aligned}
$$

where $\Omega_{0 r}, \Omega_{0 m}$ and $\Omega_{0 \phi}$ are the present energy density parameters of radiation, matter and field respectively, and 

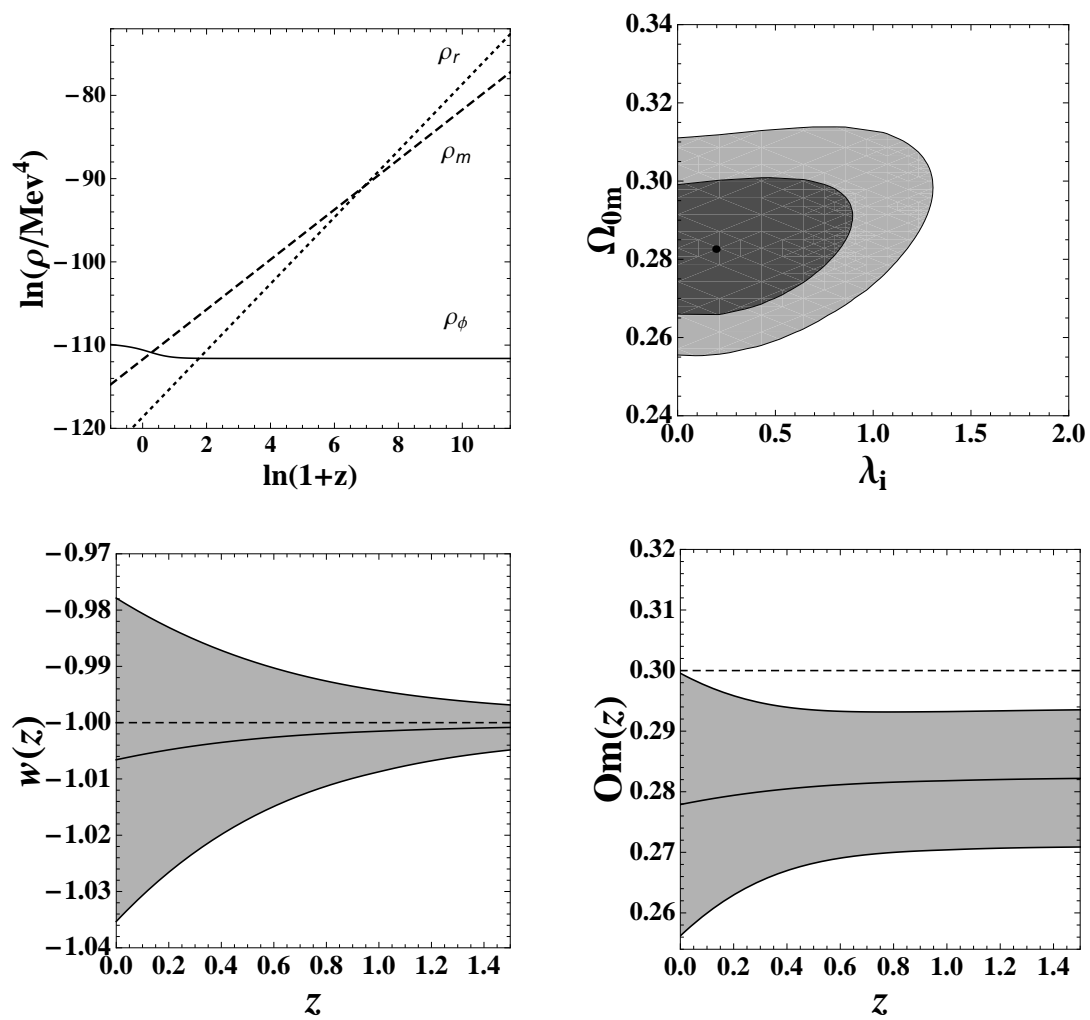

Figure 9. This figure shows different plots for the linear potential. The upper left plot shows the evolution of energy density versus the redshift $z$. The dotted, dashed and black lines correspond to the energy density of radiation, matter and phantom field respectively. Initially, the energy density of phantom field is extremely sub-dominant and remains to be so, for most of the period of evolution. At late times, the field energy density catches up with the background, overtakes it, and starts growing $(w<-1)$ and derives the current accelerated expansion of the Universe. The upper right plot shows the $1 \sigma$ (dark shaded) and $2 \sigma$ (light shaded) likelihood contours in the $\lambda_{i}-\Omega_{0 m}$ plane (the black dot designates the best fit value of $\lambda_{i}$ and $\Omega_{0 m}$, where $\lambda_{i}$ is the initial value of $\lambda$ ). The lower left and right plots show the evolution of $w(z)$ and $\operatorname{Om}(z)$ versus the redshift $z$ respectively. $\operatorname{Om}(z)$ has positive curvature. In both lower plots, the horizontal dashed line represents $\Lambda$ CDM with $\Omega_{0 m}=0.3$, solid (middle) line inside shaded regions show best fitted behaviour and shaded regions show $1 \sigma$ confidence level. We have used joint data $(\mathrm{SN}+\mathrm{Hubble}+\mathrm{BAO}+\mathrm{CMB})$ in carrying out the analysis.

$H_{0}$ designates the value of Hubble parameter at the present epoch. We will be interested in the phantom dynamics with a linear potential,

$$
V(\phi)=V_{0} \phi
$$

Numerically integrating the equations of motion, we find the field energy density, equation of state and $\mathrm{Om}$ for the said potential. The results are shown in figure 9. The upper left plot shows the evolution of energy density versus redshift $z$ whereas the upper right plot shows the $1 \sigma$ (dark shaded) and $2 \sigma$ (light shaded) likelihood contours in the $\lambda_{i}-\Omega_{0 m}$ plane. The best fit values of the parameters are found to be $\lambda_{i}=0.1959$ and $\Omega_{0 m}=0.2826$. The lower plots show the evolution of $w(z)$ and $\mathrm{Om}(z)$. As seen in the figure, $\mathrm{Om}$ has positive curvature for phantom field model which distinguishes phantom field model from zero-curvature $(\Lambda \mathrm{CDM})$, for any current value of the matter density. The joint data i.e. $\mathrm{SN}+\mathrm{Hubble}+\mathrm{BAO}+\mathrm{CMB}$ was used for analysis (see appendix A).

\section{NON MINIMALLY COUPLED SCALAR FIELD MODEL}

In this section, we revisit a non minimally coupled scalar field model that allows to obtain a transient phantom dark energy. Various features of non minimally coupled scalar field system have been investigated (Dent et al. 2013; Kamenshchik et al. 2013; Nozari \& Rashidi 2013; Aref'eva et al. 2014; Kamenshchik et al. 2014; Luo, Wu \& Yu 2014; Pozdeeva \& Vernov 2014; Skugoreva, Toporensky \& Vernov 2014; Skugoreva, Saridakis \& Toporensky 2014). The action having non minimal coupling with scalar field is given as (Sami et al. 2012):

$S=\frac{1}{2} \int \sqrt{-g} d^{4} x\left[\frac{R}{\kappa}-\left(g^{\mu \nu} \phi_{\mu} \phi_{\nu}+\xi R B(\phi)+2 V(\phi)\right)\right]+S_{M}$,

where $\kappa=8 \pi G, \xi$ is the dimensionless coupling constant and $S_{M}$ is the matter action. The equations of motion which are obtained by varying the action (30) have the form (Sami et al. 2012):

$$
H^{2}=\frac{\kappa}{3}\left(\frac{1}{2} \dot{\phi}^{2}+V(\phi)+3 \xi\left(H \dot{\phi} B^{\prime}(\phi)+H^{2} B(\phi)\right)+\rho\right),
$$



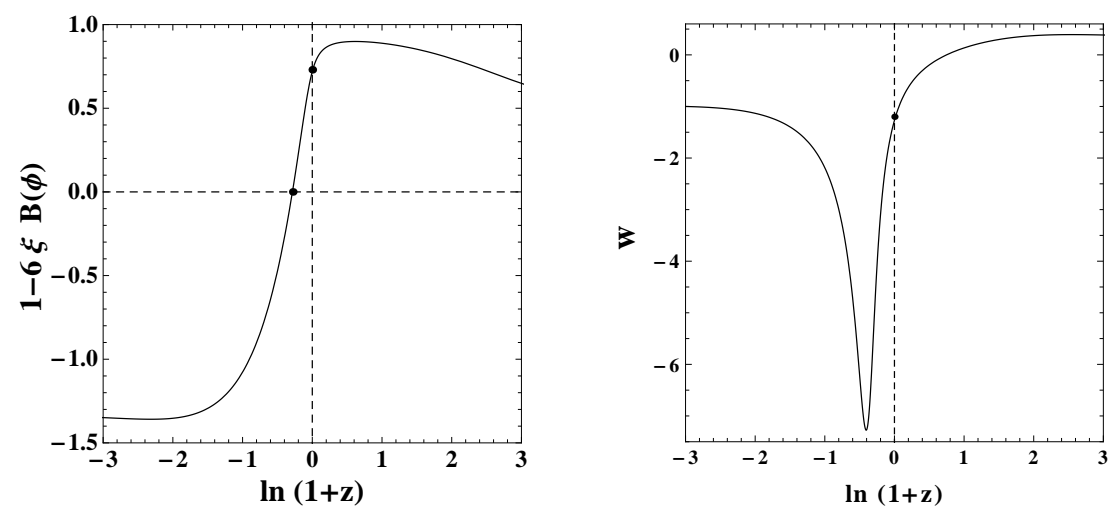

Figure 10. This figure corresponds to the case of non minimal coupling with $N=2, n=7, \xi=0.2$ and $\Omega_{0 m}=0.316$. The left and right plots show the evolution of $(1-6 \xi B(\phi))$ and w versus the redshift $z$ respectively, where $G_{e f f}=6 / 8 \pi(1-6 \xi B(\phi))$. The black dots ( point on the vertical dashed line) designate the present epoch which occurs in the regime of $G_{e f f}>0$, the effective Newtonian constant changes sign thereafter in future. The point on the horizontal dashed line is the epoch where $G_{e f f}$ turns negative.
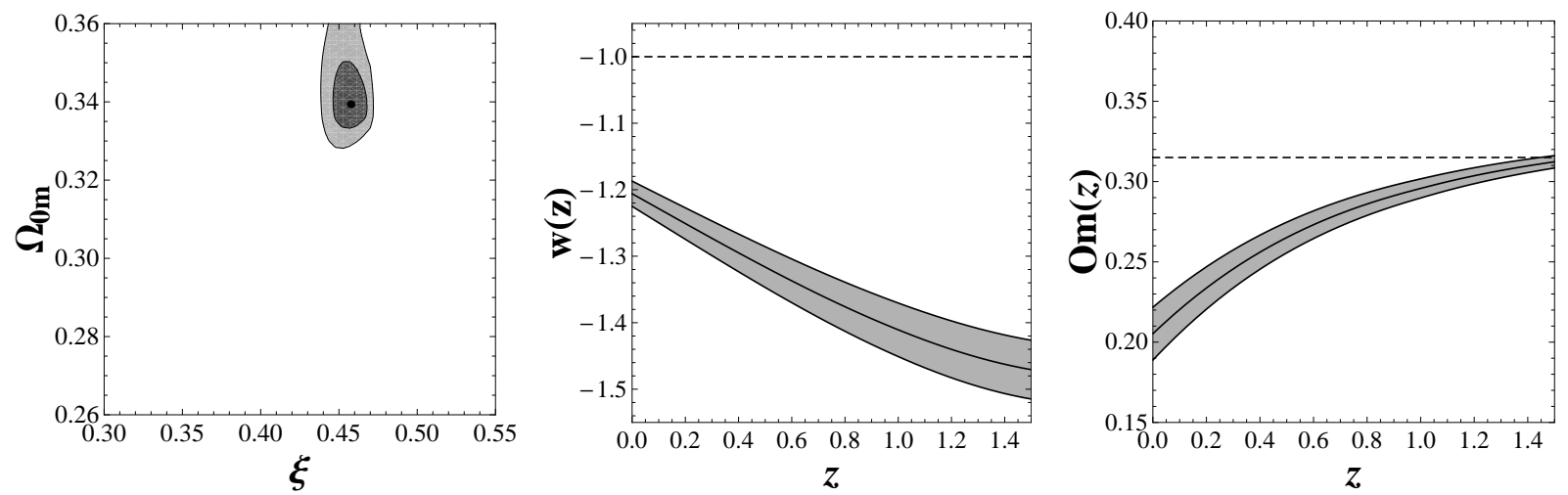

Figure 11. This figure represents the non minimally coupled scalar field DE model with $N=2, n=7$; left plot shows the $1 \sigma$ (dark shaded) and $2 \sigma$ (light shaded) likelihood contours in the $\xi-\Omega_{0 m}$ plane. The black dot corresponds to the best fit value. Evolution of $\mathrm{w}(z)$ is shown in the middle plot. The right plot shows the evolution of $O m(z)$ versus the redshift $z$. In middle and right plots, solid (middle) line inside shaded regions show best fitted behaviour and shaded regions show $1 \sigma$ confidence level; the horizontal dashed line represents $\Lambda \mathrm{CDM}$ with $\Omega_{0 m}=0.315$. The $\mathrm{SN}+\mathrm{Hubble}+\mathrm{BAO}+\mathrm{CMB}$ data has been used jointly in carrying out the analysis.

$$
\begin{gathered}
R=\kappa\left(-\dot{\phi}^{2}+4 V(\phi)+3 \xi\left(3 H \dot{\phi} B^{\prime}(\phi)+\frac{R}{3} B(\phi)\right.\right. \\
\left.\left.+\dot{\phi}^{2} B^{\prime \prime}(\phi)+\ddot{\phi} B^{\prime}(\phi)\right)+\rho(1-3 \omega)\right), \\
\ddot{\phi}+3 H \dot{\phi}+\frac{1}{2} \xi R B^{\prime}(\phi)+V^{\prime}(\phi)=0,
\end{gathered}
$$

where $R=6\left(2 H^{2}+\dot{H}\right)$, is the Ricci Scalar and $\rho$ is the energy density of the matter.

In this case (Sami et al. 2012), we have a varying effective Newtonian gravitational constant $G_{\text {eff }}$ which is a function of scalar field $\phi$. We found stationary points and their stability by considering $B(\phi)=\phi^{N}$ and $V(\phi)=V_{0} \phi^{n}$. The de Sitter solution of interest to us, exists for $n<2 N$ for which $G_{\text {eff }}=6 / 8 \pi(1-6 \xi B(\phi))=3(2 N-n) / 8 \pi N$ is positive. In order to check the stability of the de Sitter solution, we consider small perturbations, and get the system of equations (see appendix B)

$$
\left(\begin{array}{c}
\dot{\alpha} \\
\dot{\beta} \\
\dot{\gamma}
\end{array}\right)=\left(\begin{array}{ccc}
-\frac{A_{1}}{A_{2}} & -\frac{A_{3}}{A_{2}} & -\frac{A_{4}}{A_{2}} \\
0 & 0 & 1 \\
0 & -\frac{A_{5}}{A_{7}} & -\frac{A_{6}}{A_{7}}
\end{array}\right)\left(\begin{array}{l}
\alpha \\
\beta \\
\gamma
\end{array}\right)
$$

The numerical analysis of the system exhibits that the de Sitter solution is stable when $2 N+1 \leqslant n$ and for positive values of the coupling, $\xi>0$ (we assume $V_{0}=1$ ). Thus we display few cases in Table 3 which show how the nature of the fixed points depends upon numerical values of $\xi$. As demonstrated by Sami et al. (2012), the de Sitter solution in this case occurs in the region of negative effective gravitational constant, thereby leading to a ghost dominated Universe in future and a transient quintessence (phantom) phase with $G_{\text {eff }}>0$ around the present epoch. Figure 10 shows that before going to de Sitter point with $G_{e f f}<0$, the equation of state passes through a phantom phase. In order to obtain phantom $(w<-1)$ phase consistent with observation and $G_{\text {eff }}>0$ at present epoch, we adjust the model parameters as shown in figure 10. 
Table 3. Nature of the fixed points (Sami et al. 2012):

\begin{tabular}{cccc}
\hline \hline $\mathrm{N}$ & $\mathrm{n}$ & $\xi$ & nature of fixed points \\
\hline 2 & 5 & $0<\xi \leq 0.1333$ & saddle \\
& & $0.1333<\xi \leq 0.2068$ & attractive focus \\
& & $0.2069 \leq \xi \leq 1$ & attractive node \\
& & & \\
2 & 7 & $0<\xi \leq 0.0952$ & saddle \\
& & $0.0952<\xi \leq 0.3999$ & attractive focus \\
& & $0.4000 \leq \xi \leq 1$ & attractive node \\
& & & \\
2 & 9 & $0<\xi \leq 0.0740$ & saddle \\
& & $0.0740<\xi \leq 1$ & attractive focus \\
& & & \\
4 & 9 & $0<\xi<0.0009$ & saddle \\
& & $0.0009<\xi \leq 0.0014$ & attractive focus \\
& & $0.0015 \leq \xi \leq 1$ & attractive node \\
& & & \\
\hline
\end{tabular}

Let us now apply $O m$ diagnostic to the case under consideration and examine the curvature (slope) of $\mathrm{Om}$. To this effect, we investigate the evolution equations numerically in case of $N=2$ and $n=7$ for a viable range of parameters $\xi$ and $\Omega_{0 m}\left(0.24 \lesssim \Omega_{0 m} \lesssim 0.36\right.$ and $\left.0.12 \lesssim \xi \lesssim 0.61\right)$; the range of $\xi$ is dictated by stability considerations. Our results are displayed in figure 11] in which the left plot shows the $1 \sigma$ (dark shaded) and $2 \sigma$ (light shaded) likelihood contours in the $\xi-\Omega_{0 m}$ plane. The best fit values of the parameters are, $\xi=0.4574$ and $\Omega_{0 m}=0.3393$. The middle plot shows the evolution of $\mathrm{w}(z)$ versus redshift $z$ whereas the right plot displays the evolution of $\mathrm{Om}(z)$ versus redshift $z$. We find that $\mathrm{Om}$ has positive curvature (slope) for phantom equation of state which is a generic feature of dark energy models with $w<-1$. The positive curvature of phantom distinguishes non minimally coupled scalar field model from zero curvature $\Lambda$ CDM model, for any value of the matter density as shown in figure 11 We used $\mathrm{SN}+\mathrm{Hubble}+\mathrm{BAO}+\mathrm{CMB}$ joint data for carrying out the observational analysis, see appendix A for details.

\section{CONCLUSIONS}

In this paper, we have investigated scalar field models (including the phantom case) using the $O m$ diagnostic. We have specifically focused on models with power law potentials that lead to the slowing down of late time cosmic acceleration. In case of quintessence with quadratic and quartic potentials, we have demonstrated that the slowing down phenomenon takes place for $z \lesssim 0.4$. Figures 2 and 3 show that at late times, the field energy density starts decreasing $(w>-1)$ and exhibits matter/radiation like behaviour corresponding to the deceleration of expansion. Consequently, the equation of state of dark energy, $\operatorname{Om}(z)$ and the deceleration parameter $q$ grow for redshift in the interval $z \in(0,0.4)$. This signifies that cosmic acceleration might have already peaked and that we are presently observing its slowing down. We find that quintessence models have negative curvature that differentiates these models from zero curvature $(\Lambda \mathrm{CDM})$, for any given current value of the matter density as shown by the $\mathrm{Om}$ plots in figures 2 and 3 The best fit values of the model parameters for $\phi^{2}$ and $\phi^{4}$ potentials are found to be $\lambda_{i}=1.2, \Omega_{0 m}=0.2657$ and $\lambda_{i}=1.4415, \Omega_{0 m}=0.2663$ respectively. In these models, the best fit value of $\Omega_{0 m}$ is always less than that for its counter part i.e. $\Lambda$ CDM, which compensates for the effect of intermediate slowing down. We have also investigated a model with a cosh potential (19), which has the tracking property. For small values of $\phi$, the said potential mimics a power law behaviour and gives rise to oscillations of $\phi$ near the origin. The tracking behaviour of the scalar field energy density, the evolution of density parameter, the oscillating behaviour of the equation of state at late times and the corresponding behaviour of $\mathrm{Om}$ ( within $1 \sigma$ confidence level ) are shown in figures 6 and 8 The field oscillations in the convex core around the origin are reflected in the behaviour of $\mathrm{w}$ (see figure 5), such that the average equation of state parameter is given by $\langle\mathrm{w}\rangle=p-1 / p+1$ (see figure 6). The best fit values of the model parameters are, $p=0.096$, $\tilde{\alpha}=37.59, \Omega_{0 m}=0.319$. In this case, the $\Lambda \mathrm{CDM}$ is clearly outside $2 \sigma$ confidence level as shown in figure 7 . This is also clear from the figure 8 and it deserves a comment. In order to draw a final conclusion, we looked for $\chi_{\text {red }}^{2}$ and found that $\chi_{\text {red }}^{2}$ is much smaller than one. Thus we can not claim that the model under consideration is better than $\Lambda$ CDM.

Next, we applied the $O m$ diagnostic to phantom dark energy. In this case, we considered the phantom field with a linear potential. In figure 9] we have shown that $\mathrm{Om}$ has positive curvature that distinguishes the phantom dark energy from the zero curvature $\Lambda$ CDM, for any current value of the matter density. The best fit values of the model parameters are, $\lambda_{i}=0.1959, \Omega_{0 m}=0.2826$. We also examined a non minimally coupled scalar field model, which has a transient phantom behaviour. In this case again, $\mathrm{Om}(z)$ has positive curvature as shown in the figure 11. The best fit values of the model parameters are found to be $\xi=0.4574$, $\Omega_{0 m}=0.3393$. It signifies that the positive curvature of $\mathrm{Om}$ is a generic feature of phantom dark energy, be it transient or otherwise.

We conclude that given the present data, the $\mathrm{Om}$ diagnostic can clearly distinguish between scalar field models and $\Lambda \mathrm{CDM}$ and that in case of quadratic and quartic potentials, there exists a specific region in the parameter space which could allow for the slowing down of late time cosmic acceleration.

\section{ACKNOWLEDGEMENTS}

We thank S. G. Ghosh and V. Soni for their constant encouragement throughout the work. MS thanks R. Gannouji, M. W. Hossain and Sumit Kumar for useful discussions. We also thank S. Ahmad and S. Rani for helping us in improving the manuscript.

\section{APPENDIX A: OBSERVATIONAL DATA ANALYSIS}

We put constraints on the model parameters using recent observational data, namely Type Ia Supernovae, BAO, CMB and data of Hubble parameter. The total $\chi^{2}$ for joint data is defined as

$$
\chi_{\text {tot }}^{2}=\chi_{\mathrm{SN}}^{2}+\chi_{\mathrm{BAO}}^{2}+\chi_{\mathrm{Hub}}^{2}+\chi_{\mathrm{CMB}}^{2},
$$


Table 4. Values of $\frac{d_{A}\left(z_{\star}\right)}{D_{V}\left(Z_{B A O}\right)}$ for distinct values of $z_{B A O}$.

\begin{tabular}{c||cccccc}
\hline \hline$z_{B A O}$ & 0.106 & 0.2 & 0.35 & 0.44 & 0.6 & 0.73 \\
\hline$\frac{d_{A}\left(z_{\star}\right)}{D_{V}\left(Z_{B A O}\right)}$ & $30.95 \pm 1.46$ & $17.55 \pm 0.60$ & $10.11 \pm 0.37$ & $8.44 \pm 0.67$ & $6.69 \pm 0.33$ & $5.45 \pm 0.31$ \\
\hline \hline
\end{tabular}

where the $\chi_{i}^{2}$ for each data set is evaluated as follows: First, we consider the Type Ia supernova observation which is one of the direct probes for the cosmological expansion. We use Union2.1 compilation data (Suzuki et al. 2012) of 580 data points. For this case, one measures the apparent luminosity of the supernova explosion from the photon flux received. In the present context, one of the most relevant cosmological quantity is luminosity distance $D_{L}(z)$ defined as,

$$
D_{L}(z)=(1+z) \int_{0}^{z} \frac{H_{0} d z^{\prime}}{H\left(z^{\prime}\right)}
$$

Cosmologists often use the distance modulus $\mu$ defined as $\mu=m-M=5 \log D_{L}+\mu_{0}$, where $m$ and $M$ are the apparent and absolute magnitudes of the Supernovae and $\mu_{0}=5 \log \left(\frac{H_{0}^{-1}}{\mathrm{Mpc}}\right)+25$ is a nuisance parameter which is marginalized. The corresponding $\chi^{2}$ is written as

$$
\chi_{\mathrm{SN}}^{2}\left(\mu_{0}, \theta\right)=\sum_{i=1}^{580} \frac{\left[\mu_{t h}\left(z_{i}, \mu_{0}, \theta\right)-\mu_{o b s}\left(z_{i}\right)\right]^{2}}{\sigma_{\mu}\left(z_{i}\right)^{2}}
$$

where $\mu_{o b s}, \mu_{t h}$ and $\sigma_{\mu}$ represents the observed, theoretical distance modulus and uncertainty in the distance modulus respectively; $\theta$ represents any parameter of the particular model. Eventually, marginalizing $\mu_{0}$ following Lazkoz, Nesseris \& Perivolaropoulos (2005), we get

$$
\chi_{\mathrm{SN}}^{2}(\theta)=A(\theta)-\frac{B(\theta)^{2}}{C(\theta)},
$$

where,

$$
\begin{aligned}
& A(\theta)=\sum_{i=1}^{580} \frac{\left[\mu_{t h}\left(z_{i}, \mu_{0}=0, \theta\right)-\mu_{o b s}\left(z_{i}\right)\right]^{2}}{\sigma_{\mu}\left(z_{i}\right)^{2}} \\
& B(\theta)=\sum_{i=1}^{580} \frac{\mu_{t h}\left(z_{i}, \mu_{0}=0, \theta\right)-\mu_{o b s}\left(z_{i}\right)}{\sigma_{\mu}\left(z_{i}\right)^{2}} \\
& C(\theta)=\sum_{i=1}^{580} \frac{1}{\sigma_{\mu}\left(z_{i}\right)^{2}} .
\end{aligned}
$$

Next, we use BAO data of $\frac{d_{A}\left(z_{\star}\right)}{D_{V}\left(Z_{B A O}\right)}$ (Eisenstein et al. 2005; Percival et al. 2010; Beutler et al. 2011; Blake et al. 2011; Jarosik et al. 2011; Giostri et al. 2012), where $z_{\star} \approx 1091$ is the decoupling time, $d_{A}(z)=\int_{0}^{z} \frac{d z^{\prime}}{H\left(z^{\prime}\right)}$ is the co-moving angular-diameter distance and $D_{V}(z)=\left(d_{A}(z)^{2} \frac{z}{H(z)}\right)^{\frac{1}{3}}$ is the dilation scale. Data required for this analysis is shown in Table 4

The $\chi_{\mathrm{BAO}}^{2}$ is defined as (Giostri et al. 2012),

$$
\chi_{\mathrm{BAO}}^{2}=X^{T} C^{-1} X
$$

where,

$$
X=\left(\begin{array}{c}
\frac{d_{A}\left(z_{\star}\right)}{D_{V}(0.106)}-30.95 \\
\frac{d_{A}\left(z_{\star}\right)}{D_{V}(0.2)}-17.55 \\
\frac{d_{A}\left(z_{\star}\right)}{D_{V}(0.35)}-10.11 \\
\frac{d_{A}\left(z_{\star}\right)}{D_{V}(0.44)}-8.44 \\
\frac{d_{A}\left(z_{\star}\right)}{D_{V}(0.6)}-6.69 \\
\frac{d_{A}\left(z_{\star}\right)}{D_{V}(0.73)}-5.45
\end{array}\right),
$$

and $C^{-1}$ is the inverse covariance matrix given by Giostri et al. (2012).

We then use the observational data on Hubble parameter as recently compiled by Farooq \& Ratra (2013) in the redshift range $0.07 \leq z \leq 2.3$. The sample contains 28 observational data points of $H(z)$. These values are given in Table 5. To complete the data set, we take the latest and most precise measurement of the Hubble constant $H_{0}$ from PLANCK 2013 results (Ade et al. 2013). To apply the data of Hubble parameter on our models, we work with the normalized Hubble parameter, $h=H / H_{0}$.

The $\chi^{2}$ for the normalized Hubble parameter is defined as,

$$
\chi_{\mathrm{Hub}}^{2}(\theta)=\sum_{i=1}^{29} \frac{\left[h_{\mathrm{th}}\left(z_{i}, \theta\right)-h_{\mathrm{obs}}\left(z_{i}\right)\right]^{2}}{\sigma_{h}\left(z_{i}\right)^{2}},
$$

where, $h_{\mathrm{obs}}$ and $h_{\mathrm{th}}$ are the observed and theoretical values of the normalized Hubble parameter respectively.

Also,

$$
\sigma_{h}=\left(\frac{\sigma_{H}}{H}+\frac{\sigma_{H_{0}}}{H_{0}}\right) h,
$$

where $\sigma_{H}$ and $\sigma_{H_{0}}$ is the error in $H$ and $H_{0}$ respectively.

Finally, we apply $\mathrm{CMB}$ shift parameter $R=$ $H_{0} \sqrt{\Omega_{m 0}} \int_{0}^{1089} \frac{d z^{\prime}}{H\left(z^{\prime}\right)}$. The corresponding $\chi_{\mathrm{CMB}}^{2}$ can be written as,

$$
\chi_{\mathrm{CMB}}^{2}(\theta)=\frac{\left(R(\theta)-R_{0}\right)^{2}}{\sigma^{2}}
$$

where, $R_{0}=1.725 \pm 0.018$ ( Komatsu et al. 2011).

\section{APPENDIX B}

For de Sitter solution, substituting $\dot{H}=\dot{\phi}=\ddot{\phi}=\rho=0$ and $R=12 H_{0}{ }^{2}$ with $B(\phi)=\phi^{N}$ and $V(\phi)=V_{0} \phi^{n}$ in equations (31), (32) and (33), we obtain

$$
\begin{aligned}
H_{0}{ }^{2}\left(1-6 \xi \phi_{0}{ }^{N}\right) & =2 V_{0} \phi_{0}{ }^{n}, \\
6 H_{0}{ }^{2} \xi N \phi_{0}{ }^{N-1}+V_{0} n \phi_{0}{ }^{n-1} & =0,
\end{aligned}
$$

which gives

$H_{0}^{2}=-\frac{V_{0} n \phi_{0}{ }^{n-N}}{6 \xi N}, \quad \phi_{0}{ }^{N}=\frac{n}{6 \xi(n-2 N)}$.

The de Sitter solution exists provided that $n<2 N$, and $G_{\text {eff }}=6 / 8 \pi(1-6 \xi B(\phi))=3(2 N-n) / 8 \pi N$ is positive 
Table 5. $H(z)$ measurements (in unit $\left[\mathrm{km} \mathrm{s}^{-1} \mathrm{Mpc}^{-1}\right]$ ) and their errors (Farooq \& Ratra 2013).

\begin{tabular}{cccc}
\hline \hline$z$ & $H(z)$ & $\sigma_{H}$ & Reference \\
0.070 & 69 & 19.6 & Zhang et al. 2012 \\
0.100 & 69 & 12 & Simon et al. 2005 \\
0.120 & 68.6 & 26.2 & Zhang et al. 2012 \\
0.170 & 83 & 8 & Simon et al. 2005 \\
0.179 & 75 & 4 & Moresco et al. 2012 \\
0.199 & 75 & 5 & Moresco et al. 2012 \\
0.200 & 72.9 & 29.6 & Zhang et al. 2012 \\
0.270 & 77 & 14 & Simon et al. 2005 \\
0.280 & 88.8 & 36.6 & Zhang et al. 2012 \\
0.350 & 76.3 & 5.6 & Chuang \& Wang 2012 \\
0.352 & 83 & 14 & Moresco et al. 2012 \\
0.400 & 95 & 17 & Simon et al. 2005 \\
0.440 & 82.6 & 7.8 & Blake et al. 2012 \\
0.480 & 97 & 62 & Stern et al. 2010 \\
0.593 & 104 & 13 & Moresco et al. 2012 \\
0.600 & 87.9 & 6.1 & Blake et al. 2012 \\
0.680 & 92 & 8 & Moresco et al. 2012 \\
0.730 & 97.3 & 7.0 & Blake et al. 2012 \\
0.781 & 105 & 12 & Moresco et al. 2012 \\
0.875 & 125 & 17 & Moresco et al. 2012 \\
0.880 & 90 & 40 & Stern et al. 2010 \\
0.900 & 117 & 23 & Simon et al. 2005 \\
1.037 & 154 & 20 & Moresco et al. 2012 \\
1.300 & 168 & 17 & Simon et al. 2005 \\
1.430 & 177 & 18 & Simon et al. 2005 \\
1.530 & 140 & 14 & Simon et al. 2005 \\
1.750 & 202 & 40 & Simon et al. 2005 \\
2.300 & 224 & 8 & Busca et al. 2013 \\
\hline \hline & & &
\end{tabular}

only if $n<2 N$. The system of equations (31), (32) and (33) for $\rho=0$ then reduces to,

$$
\begin{aligned}
\dot{H}\left(1-6 \xi B+9 \xi^{2} B^{\prime 2}\right)= & 3 \dot{\phi}^{2}\left(\xi B^{\prime \prime}-1\right) \\
& -3 \xi B^{\prime}\left(4 H \dot{\phi}+V^{\prime}+6 \xi B^{\prime} H^{2}\right), \\
\ddot{\phi}\left(1-6 \xi B+9 \xi^{2}{B^{\prime 2}}^{2}=\right. & -\left(3 H \dot{\phi}+V^{\prime}\right)(1-6 \xi B) \\
& -\xi B^{\prime}\left(-3 \dot{\phi}^{2}+12 V+9 \xi\left(3 H \dot{\phi} B^{\prime}\right.\right. \\
& \left.\left.+\dot{\phi}^{2} B^{\prime \prime}\right)\right) .
\end{aligned}
$$

To check the stability of the de Sitter, we consider small perturbations $\mu \& \nu$ around this background: $H=H_{0}+\mu$ and $\phi=\phi_{0}+\nu$ in the dynamical system (49) which gives us the evolution equations for perturbations

$$
\begin{aligned}
& \mu\left(36 \xi^{2} N^{2} H_{0}{\phi_{0}}^{2 N-2}\right) \\
& +\dot{\mu}\left(1-6 \xi \phi_{0}{ }^{N}+9 \xi^{2} N^{2} \phi_{0}{ }^{2 N-2}\right) \\
& +\nu\left(3 \xi V_{0} N n(N+n-2) \phi_{0}{ }^{N+n-3}\right. \\
& \left.+36 \xi^{2} N^{2}(N-1) H_{0}{ }^{2} \phi_{0}{ }^{2 N-3}\right) \\
& +\dot{\nu}\left(12 \xi N H_{0} \phi_{0}{ }^{N-1}\right)=0 \\
& \nu\left(V_{0} n(n-1) \phi_{0}{ }^{n-2}\left(1-6 \xi \phi_{0}{ }^{N}\right)\right. \\
& \left.-6 \xi V_{0} N n \phi_{0}{ }^{N+n-2}+12 \xi V_{0} N(N+n-1) \phi_{0}{ }^{N+n-2}\right) \\
& +\dot{\nu}\left(3 H_{0}\left(1-6 \xi \phi_{0}{ }^{N}\right)+27 \xi^{2} N^{2} H_{0} \phi_{0}{ }^{2 N-2}\right) \\
& +\ddot{\nu}\left(1-6 \xi \phi_{0}{ }^{N}+9 \xi^{2} N^{2} \phi_{0}{ }^{2 N-2}\right)=0
\end{aligned}
$$

Equations (50) and (51) can be put in a simple form by introducing the following notations

$$
\begin{aligned}
A_{1}= & 36 \xi^{2} N^{2} H_{0} \phi_{0}{ }^{2 N-2}, \\
A_{2}= & 1-6 \xi \phi_{0}{ }^{N}+9 \xi^{2} N^{2} \phi_{0}{ }^{2 N-2}, \\
A_{3}= & 3 \xi V_{0} N n(N+n-2) \phi_{0}{ }^{N+n-3} \\
& +36 \xi^{2} N^{2}(N-1) H_{0}{ }^{2} \phi_{0}{ }^{2 N-3}, \\
A_{4}= & 12 \xi N H_{0} \phi_{0}{ }^{N-1}, \\
A_{5}= & V_{0} n(n-1) \phi_{0}{ }^{n-2}\left(1-6 \xi \phi_{0}{ }^{N}\right)-6 \xi V_{0} N n \phi_{0}{ }^{N+n-2} \\
& +12 \xi V_{0} N(N+n-1) \phi_{0}{ }^{N+n-2}, \\
A_{6}= & 3 H_{0}\left(1-6 \xi \phi_{0}{ }^{N}\right)+27 \xi^{2} N^{2} H_{0} \phi_{0}{ }^{2 N-2}, \\
A_{7}= & 1-6 \xi \phi_{0}{ }^{N}+9 \xi^{2} N^{2} \phi_{0}{ }^{2 N-2}
\end{aligned}
$$

Next, by using, $\alpha=\mu, \beta=\nu, \gamma=\dot{\nu}$, the system of equations (50) and (51) acquires a simple form,

$$
\begin{aligned}
& A_{1} \alpha+A_{2} \dot{\mu}+A_{3} \beta+A_{4} \gamma=0, \\
& A_{5} \beta+A_{6} \gamma+A_{7} \ddot{\nu}=0 .
\end{aligned}
$$

Taking derivative of $\alpha, \beta$ and $\gamma$ with respect to time we get the system of equations,

$$
\left(\begin{array}{c}
\dot{\alpha} \\
\dot{\beta} \\
\dot{\gamma}
\end{array}\right)=\left(\begin{array}{ccc}
-\frac{A_{1}}{A_{2}} & -\frac{A_{3}}{A_{2}} & -\frac{A_{4}}{A_{2}} \\
0 & 0 & 1 \\
0 & -\frac{A_{5}}{A_{7}} & -\frac{A_{6}}{A_{7}}
\end{array}\right)\left(\begin{array}{c}
\alpha \\
\beta \\
\gamma
\end{array}\right)
$$

\section{REFERENCES}

Ade P. A. R. et al., 2013, arXiv:1303.5076] [astro-ph.CO] Alam U., Sahni V., Starobinsky A. A., 2003, J. Cosmol. Astropart. Phys. 04002

Alam U., Sahni V., Saini T. D., Starobinsky A. A., 2003, MNRAS 344, 1057

Ali A., Gannouji R., Sami M., 2010, Phys. Rev. D 82 103015

Arabsalmani M., Sahni V., 2011, Phys. Rev. D 83043501

Aref'eva I. Y., Bulatov N. V., Gorbachev R.V., Vernov S. Y., 2014, Class. Quant. Grav. 31065007

Beutler F. et al., 2011, Mon. Not. Roy. Astron. Soc. 416 3017

Blake C. et al., 2011, Mon. Not. Roy. Astron. Soc. 4181707 Blake C. et al., 2012, Mon. Not. Roy. Astron. Soc. 425405 Boisseau B., Esposito-Farese G., Polarski D., Starobinsky A. A., 2000, Phys. Rev. Lett. 852236

Brevik I., 2008 Eur. Phys. J. C 56579

Busca N. G. et al., 2013, Astron. Astrophys. 552 A96

Caldwell R. R., Kamionkowski M., Weinberg N. N., 2003,

Phys. Rev. Lett. 91, 071301

Chuang C. H., Wang Y., 2013, Mon. Not. Roy. Astron. Soc. 435 255-262, arXiv:1209.0210 [astro-ph.CO]

Copeland E. J., Sami M., Tsujikawa S., 2006, Int. J. Mod. Phys. D 15, 1753

Dent J. B., Dutta S., Saridakis E. N., Xia J. Q., 2013, JCAP 1311058

Dvali G., Gabadadze G., Porrati M., 2000 Phys. Lett. B 485208

Eisenstein D. J. et al., 2005, Astrophys. J. 633, 560

Farooq O., Ratra B., 2013, Astrophys. J. 766 L7

Felder G., Frolov A., Kofman L., Linde A., 2002, Phys. Rev. D 66023507 
Frampton P. H., Takahashi T., 2003 Phys. Lett. B 557 135138

Frieman J., Turner M., Huterer D., 2008, Ann. Rev. Astron .Astrophys. 46 385-432

Gannouji R., Sami M., 2010, Phys. Rev. D 82024011

Giostri R., Santos M. V. d., Waga I., Reis R. R. R., Calvao M. O., Lago B. L., 2012, JCAP 03027

Jamil M., Momeni D., Myrzakulov R., 2013, Eur. Phys. J. C 732347

Jamil M., Momeni D., Myrzakulov R., Rudra P., 2012, J. Phys. Soc. Jpn., Vol.81, No.11, p.114004

Jarosik N. et al., 2011, Astrophys. J. Suppl. 19214

Kallosh R., Linde A., 2003, J. Cosmol. Astropart. Phys. 02 002

Kallosh R., Linde A., Prokushkin S., Shmakova M., 2002, Phys. Rev. D 66123503

Kamenshchik A. Y., Pozdeeva E. O., Tronconi A., Venturi G., Vernov S. Y., 2014, Class.Quant.Grav. 31105003

Kamenshchik A. Y., Tronconi A., Venturi G., Vernov S. Y., 2013, Phys. Rev. D 87063503

Komatsu E. et al., 2011, ApJS, 192, 18

Krauss L. M., Chaboyer B., 2003, Science 299 65-70

Lazkoz R.,Nesseris S., Perivolaropoulos L., 2005, JCAP 0511010

Li J., Yang R. J., Chen B., 2014, arXiv:1406.7514

Luo X., Wu P., Yu H., 2014, Astrophys.Space Sci. 350 831837

Moresco M., Verde L., Pozzetti L., Jimenez R., Cimatti A., 2012, JCAP 07053

Myrzakulov R., Shahalam M., 2013, J. Cosmol. Astropart. Phys. 10 047, arXiv:1303.0194

Myrzakulov R., Shahalam M., 2014, arXiv:1407.7798.

Nozari K., Rashidi N., 2013, Astrophys.Space Sci. 347 375388

Percival W. J. et al., 2010, Mon. Not. Roy. Astron. Soc. 4012148

Perlmutter S. et al., 1999, Astrophys.J. 517, 565

Pozdeeva E. O., Vernov S. Y., 2014, arXiv:1401.7550

Rani S., Altaibayeva A., Shahalam M., Singh J. K., Myrzakulov R., 2014, arXiv:1404.6522.

Ratra B., Peebels P.J.E., 1988,Phys. Rev. D. 373406

Riess A. G., et al., 1998, Astron. J. 116, 1009

Sahni V., Saini T. D., Starobinsky A. A., Alam U., 2003, jetpl 77, 201

Sahni V., Shafieloo A., Starobinsky A. A., 2008, Phys. Rev. D 78103502

Sahni V., Shafieloo A., Starobinsky A. A., 2014, Astrophys. J. 793 L40, arXiv:1406.2209.

Sahni V., Shtanov Yu., 2003 J. Cosmol. Astropart. Phys. 11014

Sahni V., Shtanov Yu., Viznyuk A., 2005 J. Cosmol. Astropart. Phys. 12005

Sahni V., Starobinsky A. A., 2000, Int. J. Mod. Phys. D 9, 373

Sahni V., Wang L., 2000, Phys. Rev. D 62103517

Sami M., 2009, Curr. Sci. 97, 887 arXiv:0904.3445

Sami M., Myrzakulov R., 2013 arXiv:1309.4188.

Sami M., Shahalam M., Skugoreva M., Toporensky A., 2012, Phys. Rev. D 86, 103532

Setare M. R., 2007, Eur. Phys. J. C 50991

Shafieloo A., Sahni V., Starobinsky A. A., 2009, Phys. Rev. D 80101301
Shafieloo A., Sahni V., Starobinsky A. A., 2012, Phys. Rev. D 86, 103527

Simon J., Verde L., Jimenez R., 2005, Phys. Rev. D 71 123001

Singh P., Sami M., Dadhich N., 2003, Phys.Rev. D 68 023522

Skugoreva M. A., Toporensky A. V., Vernov S. Y., 2014, Phys. Rev. D 90, 064044

Skugoreva M., Saridakis E., Toporensky A., 2014, arXiv:1412.1502

Spergel D. N. et al., 2003, Astrophys. J. Suppl. 148175

Stern D., Jimenez R., Verde L., Kamionkowski M., Stanford S. A., 2010, JCAP 1002008

Suzuki N. et al., 2012, Astrophys. J. 74685

Trodden M., 2007, Int. J. Mod. Phys. D 162065

Weinberg S., 1989, Mod. Phys. Rev. 61527

Zhang J. F., Cui J. L. and Zhang X., 2014, arXiv:1409.6562 [astro-ph.CO]

Zhang C., Zhang H., Yuan S., Zhang T. Z., Sun Y. C., 2012, arXiv:1207.4541 [astro-ph.CO]

Zunckel C., Clarkson C., 2008, Phys. Rev. Lett. 101, 181301, arXiv:0807.4304 [astro-ph]]. 